INVESTIGACIONES

\title{
Factores de aula asociados al desarrollo integral de los estudiantes: Un estudio observacional
}

\author{
Teaching factors associated with the comprehensive development of students: \\ An observational study
}

\author{
F. Javier Murillo ${ }^{a}$ Cynthia Martínez Garrido \\ a Universidad Autónoma de Madrid. \\ Correo electrónico: javier.murillo@uam.es \\ ${ }^{\mathrm{b}}$ Universidad Autónoma de Madrid. \\ Correo electrónico: cynthialme@gmail.com.
}

\begin{abstract}
RESUMEN
Este artículo presenta el proceso y los resultados de una investigación que busca identificar los factores de aula asociados al desarrollo integral de los estudiantes de Iberoamericanos de Educación Primaria. Para realizarlo, investigadores educativos observan 254 aulas de 98 escuelas en nueve países de Iberoamérica con un guión común, y lo relacionan con el rendimiento en matemáticas, el autoconcepto y la satisfacción con la escuela de los 5.603 estudiantes que asisten a esas aulas. Los resultados apuntan a factores tales como clima del aula, preparación y estructuración de las clases, actividades didácticas desarrolladas, evaluación y retroalimentación, tareas para casa, y gestión del tiempo del aula son factores asociados al rendimiento, autoconcepto y bienestar de los estudiantes en la escuela. Todo ello permite conocer mejor los factores que inciden en el desarrollo de los estudiantes.
\end{abstract}

Palabras Clave: Enseñanza Eficaz, Observación, Educación Primaria, Modelos Multinivel, Iberoamérica.

\section{ABSTRACT}

This paper shows classroom factors related to the integral development of Ibero-American students in primary education. It presents a study in which educational researchers observed 254 classrooms at 98 schools in 9 IberoAmerican countries. The results show the relationship between the data from observation process and student mathematics performance, self-concept, and school satisfaction. The data describe classroom factors associated with student' performance, self-concept, and school wellbeing. These factors include classroom environment, lesson preparation and structure, activities, assessment and feedback, homework, and classroom time management. Our results allow a greater comprehension of the classroom factors that impact student development

Keywords: Effective Teaching, Observation, Primary Education, Multilevel Models, Ibero-America. 


\section{INTRODUCCIÓN}

La investigación sobre Enseñanza Eficaz (Teaching Effectiveness Research) ha aportado una gran cantidad de información sobre los factores de aula que determinan el desempeño de los estudiantes. Sin embargo, la mayoría de los estudios desarrollados hasta el momento tienen dos importantes limitaciones. De un lado, son estudios basados en cuestionarios, de tal forma que se poseía información de la autopercepción de lo que hace el docente o, en algunos casos, de lo que dice el estudiante sobre ello. Extremadamente infrecuentes son estudios de carácter cuantitativo, y más con grandes muestras, donde la información es obtenida mediante una observación sistemática realizada por el investigador. La segunda limitación de estos trabajos es que utilizan medidas de producto de carácter exclusivamente académico como, por ejemplo, el rendimiento académico de los estudiantes en pruebas estandarizadas. Aunque existen interesantes estudios sobre el trabajo con factores socioafectivos, aún tenemos poca información al respecto.

Con esta investigación pretendemos tener más información sobre los factores de aula que inciden en el desarrollo de los estudiantes intentando superar ambas limitaciones: de un lado, usando la observación sistemática y, por otro, trabajando no sólo con medidas de rendimiento cognitivo, también socio-afectivo.

\section{MARCO TEÓRICO}

Desde los años 60, la investigación sobre enseñanza eficaz ha aportado una gran cantidad de información sobre aquellos factores de aula que inciden en el desarrollo de los estudiantes (Marzano 2003; Murillo, Martínez-Garrido y Hernández-Castilla 2011; Wittrock 1986). En esencia, nueve son los grandes factores de eficacia de aula según la literatura internacional.

En primer lugar, hay que señalar el Clima de aula como uno de los factores de enseñanza eficaz (Murillo y Martínez-Garrido 2012). Según la investigación (Thapa et al. 2013), las aulas donde los niños y niñas aprenden más es aquella donde se sienten queridos y valorados por parte de sus docentes, hay ausencia de ausencia de violencia física y verbal, así como de situaciones de discriminación y marginación, definido por un ambiente ordenado y centrado en el trabajo. El segundo de los factores es la preparación de las clases. Según los investigadores el tiempo que el docente dedica a preparar las sesiones, así como los materiales que usa para ello, son un prerrequisito necesario para lograr una enseñanza eficaz que define la calidad de los docentes. Hasta tal punto que Gran, Hindman y Stronge señalaron, quizá de una forma un tanto exagerada, que era el responsable del 52\% del éxito de los estudiantes de primaria en Matemáticas en la Etapa Primaria (Hindman y Stronge 2010). Gamoran y sus colaboradores, por su parte, encontraron que una adecuada planificación de los contenidos por parte del docente contrarresta hasta en un $20 \%$ el efecto negativo que causa la falta de recursos en el aula (Gamoran y colaboradores 1997).

Otro factor que la investigación señala que marca diferencias es el llamado "lecciones estructuradas" (p.e. Muijs y Reynolds 2001). Como tal se entiende que el o la docente formula objetivos para cada lección, considera los conocimientos previos, plantea contenidos, actividades y estrategias de evaluación; y comunica todo ello a los estudiantes al inicio de cada lección (Hunt, Wiseman y Touzel 2009). No cabe duda de que las actividades que realiza el docente en el aula tienen una influencia determinante en el aprendizaje de 
los estudiantes. La investigación demuestra que, dependiendo del estudiante, de la materia, del momento y del docente, será más eficaz el uso de una metodología que otra, o la combinación de varias (Borich 2009). Algunos elementos clave que garantizan el desarrollo de los estudiantes son: a) realizar actividades variadas (Hunt, Wiseman y Touzel 2009), b) fomentar la participación de los estudiantes (Killen 2006), y c) donde estén implicados activamente (Muijs y Reynolds 2001).

Sólidas evidencias científicas consideran la evaluación del estudiante como factor directamente ligado a una docencia de calidad (Brookhart 2009; Murillo, Martínez-Garrido e Hidalgo 2014). La evaluación impacta sobre el rendimiento de los estudiantes si se utilizan variadas estrategias de evaluación y si los resultados de ésta se utilizan con el fin de reforzar y retroalimentar el proceso de aprendizaje de los estudiantes (Orlich et al. 2010). Un sexto factor es el de tareas para casa. Un docente eficaz considera las tareas para casa como una manera de afianzar conocimientos en los estudiantes, responder a la heterogeneidad del aula y comprobar el desarrollo alcanzado por ellos (Trautwein y Köller 2003). Aunque el efecto de las tareas para casa sobre el rendimiento de los estudiantes no es necesariamente mayor cuanto mayor sea la dedicación (Cooper 2007) es el estudio del tiempo dedicado a realizarlas el que más investigaciones ha concentrado (Murillo y Martínez-Garrido 2013).

La gestión del tiempo delimita la optimización de las oportunidades para aprender. Una de las cuestiones críticas de las aulas es la pérdida de oportunidades de aprendizaje (Borich 2009). Se logra maximizar las oportunidades de aprendizaje cuando las sesiones son puntuales; se minimice el tiempo para la organización de la clase; se maximice el tiempo dedicado a enseñar; y cuándo los estudiantes están motivados (Martínez-Garrido 2015).

Junto a ellos, se encuentran las altas expectativas (Rubie-Davies 2010), y la satisfacción, implicación y compromiso del docente (Thijs y Verkuyterm 2009). Estos dos últimos, sin embargo, son de difícil observación directa y, por lo tanto, no son incluidos en esta investigación.

La investigación sobre enseñanza eficaz y eficacia escolar en Iberoamérica, tiene, como señala Murillo, una larga tradición habiéndose publicado algunos trabajos de excelente calidad (Murillo 2007a). Una revisión de los mismos (cuadro 1), indica que los factores antes señalados también han aparecido en ellos. 
Cuadro 1. Factores de enseñanza eficaz en la investigación iberoamericana

\begin{tabular}{|c|c|c|c|c|c|c|c|}
\hline & 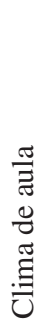 & 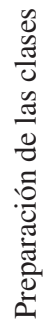 & 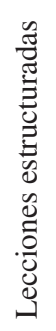 & 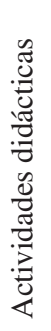 & 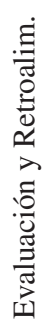 & $\begin{array}{l}\tilde{J} \\
\tilde{J} \\
\widetilde{J} \\
\tilde{\Xi} \\
\tilde{\Xi} \\
\tilde{J} \\
\tilde{J}\end{array}$ & 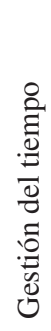 \\
\hline Muñoz-Izquierdo et al. (1979) & & & & $\mathrm{X}$ & & & \\
\hline Villa (1983) & $\mathrm{X}$ & & $\mathrm{X}$ & & & & \\
\hline López, Assael y Neuman (1984) & $\mathrm{X}$ & & $\mathrm{X}$ & $\mathrm{X}$ & & & \\
\hline Fuentes (1995) & $\mathrm{X}$ & $\mathrm{X}$ & $\mathrm{X}$ & $\mathrm{X}$ & $\mathrm{X}$ & & $\mathrm{X}$ \\
\hline Concha (1996) & $\mathrm{X}$ & & & $\mathrm{X}$ & & & $\mathrm{X}$ \\
\hline Schiefelbein, Vélez y Valenzuela (1997) & $\mathrm{X}$ & $\mathrm{X}$ & $\mathrm{X}$ & $\mathrm{X}$ & $\mathrm{X}$ & $\mathrm{X}$ & $\mathrm{X}$ \\
\hline Carreras, Guil y Mestre (1999) & $\mathrm{X}$ & & & & $\mathrm{X}$ & & \\
\hline Cueto et al. (2002) & & & & $\mathrm{X}$ & & $\mathrm{X}$ & $\mathrm{X}$ \\
\hline Román (2003) & $\mathrm{X}$ & & & $\mathrm{X}$ & $\mathrm{X}$ & $\mathrm{X}$ & \\
\hline Fernández Aguerre (2004) & & & $\mathrm{X}$ & & & & $\mathrm{X}$ \\
\hline López (2006) & $\mathrm{X}$ & $\mathrm{X}$ & $\mathrm{X}$ & $\mathrm{X}$ & $X$ & & \\
\hline Murillo (2006) & $\mathrm{X}$ & $\mathrm{X}$ & & $\mathrm{X}$ & $\mathrm{X}$ & $\mathrm{X}$ & \\
\hline Sancho (2006) & $\mathrm{X}$ & $\mathrm{X}$ & $\mathrm{X}$ & $\mathrm{X}$ & & $\mathrm{X}$ & \\
\hline Cornejo y Redondo (2007) & $\mathrm{X}$ & & $\mathrm{X}$ & $\mathrm{X}$ & $\mathrm{X}$ & $\mathrm{X}$ & $\mathrm{X}$ \\
\hline Murillo (2007b) & $\mathrm{X}$ & $\mathrm{X}$ & & $\mathrm{X}$ & & & $\mathrm{X}$ \\
\hline Duarte, Bos y Moreno (2011) & & & $\mathrm{X}$ & & & & $\mathrm{X}$ \\
\hline Román y Murillo (2011) & $\mathrm{X}$ & & $\mathrm{X}$ & & & & \\
\hline Hernández-Castilla, Murillo y Martínez-Garrido (2014) & $\mathrm{X}$ & & $\mathrm{X}$ & $\mathrm{X}$ & $\mathrm{X}$ & & $\mathrm{X}$ \\
\hline
\end{tabular}

Fuente: Elaboración propia.

Además, esta enseñanza eficaz se ve influida y definida tanto por las características, valores y conocimientos del docente (Adegbile y Adeyemi 2008) como por el contexto de escuela, político y social en el que se desarrolla su trabajo (Darling-Hammond 2000). Con todo ello, en el Gráfico 1 se tiene una imagen global del marco teórico de la presente investigación. 
Gráfico 1. Marco global de la investigación (Fuente: Elaboración propia)

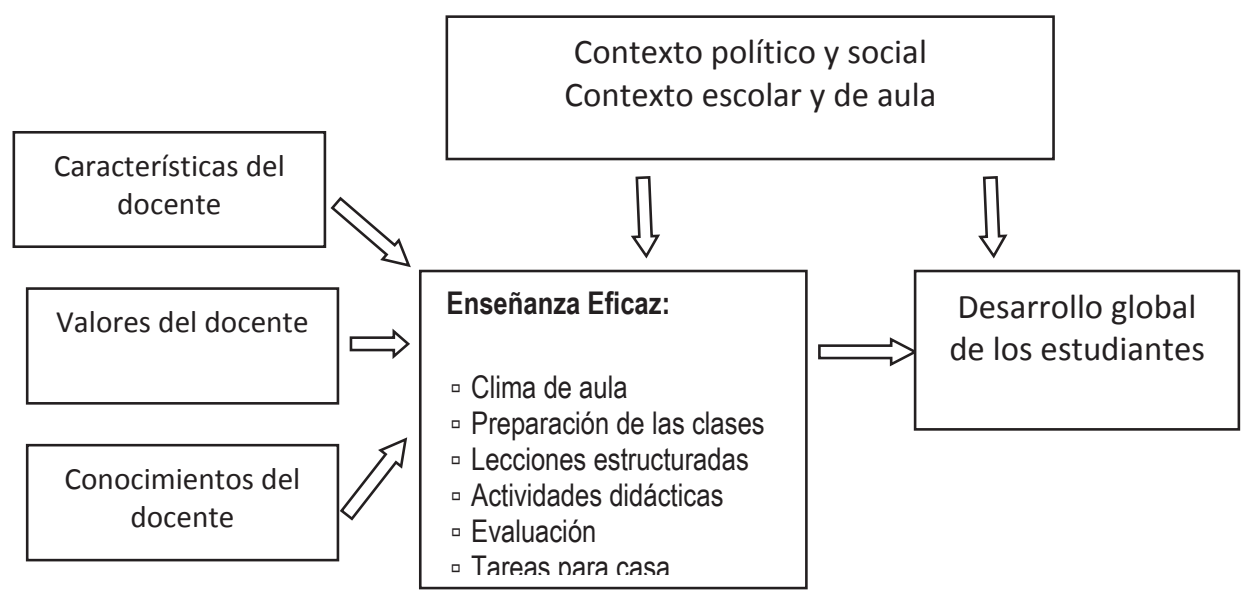

Con esta investigación se pretende Identificar los factores de aula asociados al desarrollo integral de los estudiantes de Iberoamericanos de Educación Primaria. Para ello se realiza un estudio ex-post facto de carácter observacional, mediante observación sistemática de las prácticas del aula por investigadores, y se contrastan los resultados con tres variables de producto controladas por diferentes variables de ajuste, mediante Modelos Multinivel de cuatro niveles de análisis (estudiante, aula, escuela y país).

\section{MÉTODO}

Concretamente se estudiaron las siguientes variables:

- Variables de producto:

- Desempeño de los estudiantes en Matemáticas estimado mediante Teoría de Respuesta al Ítem, media de 250 y desviación típica de 50.

- Autoconcepto, percepción que cada persona tiene de sí misma, elaborada a través de lo que piensa o sabe de cómo es y de cómo lo ven los demás. Se presenta con una media de 250 y una desviación típica de 50.

- Satisfacción del estudiante con la escuela, valoración global de los estudiantes sobre su escuela.

- Variables contextuales de ajuste:

- Rendimiento previo en Matemáticas, obtenido a través de una prueba de rendimiento al comienzo del curso;

- Nivel socio-económico de la familia del estudiante, variable tipificada, obtenida a partir de la profesión de los padres y las posesiones familiares;

- Nivel cultural de la familia del estudiante, obtenida como promedio de la titulación máxima conseguida por ambos padres, tipificada; 
- Género, variable dicotómica;

- Lengua materna, si la lengua materna del estudiante es el castellano u otra, variable dummy; $\mathrm{y}$

○ Origen, si el estudiante ha nacido o no en el país donde estudia, variable dummy.

- Variables de proceso del aula aparecen en el cuadro 2.

Cuadro 2. Variables de proceso analizadas

\begin{tabular}{|c|c|}
\hline Clima & $\begin{array}{l}\text { Relación entre los alumnos: interacciones tanto académicas como no. } \\
\text { Relación entre alumnos y profesor: interacciones tanto académicas como no. } \\
\text { Contacto físico con los alumnos positivo: consideración, cariño, apoyo, protección, etc. } \\
\text { Comunicación positiva entre los alumnos: intercambio constructivo de opiniones, ideas } \\
\text { y experiencias. } \\
\text { Ambiente orientado al aprendizaje: las actividades contribuyen al proceso de enseñanza- } \\
\text { aprendizaje. } \\
\text { Molestias mutuas entre los alumnos: interrupciones de unos estudiantes a otros. } \\
\text { Calidez (verbal) del profesor hacia los alumnos: cariño, preocupación, protección, etc. } \\
\text { del profesor hacia los alumnos. }\end{array}$ \\
\hline $\begin{array}{l}\text { Preparación de las } \\
\text { clases }\end{array}$ & $\begin{array}{l}\text { Dominio del contenido de enseñanza: el profesor expresa los contenidos con rigor } \\
\text { científico, sin cometer imprecisiones o errores. } \\
\text { Planificación de la secuencia didáctica: el docente dispone de plan mental para desarrollar } \\
\text { las actividades de clase. }\end{array}$ \\
\hline & $\begin{array}{l}\text { Aseguramiento del nivel de partida: el profesor busca la activación de conocimientos } \\
\text { previos. } \\
\text { Explicación de los objetivos desde el inicio: las explicaciones del profesor al inicio de la clase. } \\
\text { Consolidación de un punto antes de avanzar: de forma sistemática, el profesor resume y } \\
\text { refuerza lo trabajado y verifica su comprensión. } \\
\text { Recordatorio sistemático de los objetivos: el profesor valora el cumplimiento parcial de } \\
\quad \text { los objetivos. } \\
\text { Síntesis de los contenidos al finalizar la sesión: al finalizar la sesión, el presenta una } \\
\quad \text { síntesis conclusiva de los contenidos. }\end{array}$ \\
\hline & $\begin{array}{l}\text { Técnicas de indagación: diálogos entre el profesor y los alumnos tendentes a propiciar el } \\
\text { proceso de enseñanza-aprendizaje. } \\
\text { Resolución de problemas: se plantea a los alumnos situaciones nuevas que éstos deben } \\
\text { resolver. } \\
\text { Desarrollo del pensamiento de los estudiantes: orientado al logro de un aprendizaje } \\
\text { significativo. } \\
\text { Ajuste de los problemas y tareas al contenido: a la naturaleza de los contenidos } \\
\text { trabajados. } \\
\text { Vinculación del contenido de enseñanza con la vida: en las actividades se pone especial } \\
\text { énfasis en su funcionalidad y aplicabilidad. } \\
\text { Estimulación del pensamiento crítico y creativo: en la clase se exige una elaboración } \\
\text { crítica y creativa de los contenidos por parte de los alumnos. } \\
\text { Insistencia en la colaboración: hincapié en la colaboración entre estudiantes. } \\
\text { Fomento de los buenos modales: durante la clase fomenta un estilo de comportamiento } \\
\text { acorde con los patrones de convivencia social admitidos. } \\
\text { Consolidación de valores patrióticos y humanistas: se promueven reflexiones de carácter } \\
\text { patriótico y/o morales. }\end{array}$ \\
\hline yentación & $\begin{array}{l}\text { Refuerzo: el profesor emplea el reconocimiento de los éxitos y las aportaciones de los } \\
\text { alumnos. } \\
\text { Castigo: el profesor emplea la reprimenda o el castigo con el fin de modificar la conducta } \\
\text { perturbadora de los alumnos. } \\
\text { Retroalimentación y corrección informativas: el docente aporta nuevos elementos } \\
\text { esclarecedores durante la corrección y el repaso. }\end{array}$ \\
\hline
\end{tabular}




\begin{tabular}{|l|l|}
\hline Tareas para casa & $\begin{array}{l}\text { Calidad de su orientación: el profesor ofrece recomendaciones generales para realizarlas. } \\
\text { Control de la ejecución: el profesor recoge información acerca de la realización o no de } \\
\text { las tareas para casa. }\end{array}$ \\
\hline $\begin{array}{l}\text { Gestión del } \\
\text { tiempo }\end{array}$ & $\begin{array}{l}\text { Distribución del tiempo en la clase: la sesión se desarrolla completa, no se observan } \\
\text { pérdidas de tiempo. } \\
\text { Concesión de tiempo para que los alumnos piensen: el profesor realiza pautas para que } \\
\text { los alumnos respondan a las peticiones que éste realiza. } \\
\text { Puntualidad de los alumnos: en la llegada a clase, en las entradas de los recreos... se } \\
\text { observa que no se demoran. }\end{array}$ \\
\hline
\end{tabular}

Fuente: Elaboración propia.

La muestra estudiada está conformada por 5.603 estudiantes de 248 aulas de tercer curso de Primaria de 98 escuelas de nueve países de Iberoamérica (Bolivia, Chile, Colombia, Cuba, Ecuador, España, Panamá, Perú y Venezuela). Como criterio general se planteó estudiar diez escuelas públicas de cada país en las que se impartiera Educación Básica/Primaria. Sin embargo, Chile, Colombia y Venezuela obtuvieron datos de nueve escuelas; Ecuador analizó 11 escuelas; y Perú, decidió ampliar el número de escuelas a 20 (cuadro 3). Análisis previos realizados indican que esta ampliación de la muestra de Perú, no altera los resultados.

Para su selección, se utilizaron dos criterios jerarquizados: a) maximizar la varianza experimental. Para ello se intentó seleccionar cuatro escuelas de alto desempeño, tres de bajo desempeño y las tres restantes de medio desempeño; y b) obtener la máxima representatividad ecológica, se utilizaron tres subcriterios: (i) que las escuelas reflejasen la variedad regional del país; (ii) hábitat de la localidad donde están ubicadas: megaciudad, zona urbana y zona rural, y (iii) tamaño de las escuelas, se analizaron escuelas grandes, medianas y pequeñas, en función del tamaño medio del país.

Cuadro 3. Muestra del estudio

\begin{tabular}{|l|c|c|c|}
\hline & ESCUELAS & Aulas & EstudianTES \\
\hline Bolivia & 10 & 30 & 665 \\
\hline Chile & 9 & 17 & 407 \\
\hline Colombia & 9 & 20 & 465 \\
\hline Cuba & 10 & 37 & 678 \\
\hline Ecuador & 11 & 26 & 678 \\
\hline España & 10 & 21 & 335 \\
\hline Panama & 10 & 26 & 447 \\
\hline Perú & 20 & 48 & 1.566 \\
\hline Venezuela & 9 & 23 & 362 \\
\hline Total & 98 & 248 & 5.603 \\
\hline
\end{tabular}

Fuente: Elaboración propia. 
Respecto a las aulas, se decidió estudiar los grupos de tercer grado de Educación Primaria/Básica (8/9 años de edad modal). De esta forma, se seleccionaron todas las aulas de tercer grado de las escuelas ya elegidas. En caso de que alguna de ellas tuviera más de cinco aulas de ese grado, se eligieron cinco al azar. Por último, se pretendió estudiar todos los niños y niñas que asisten a las aulas seleccionadas.

Una vez seguidas estas consideraciones, la muestra final de nuestro estudio la conforman 5.603 estudiantes; es decir, todos aquellos de los que se obtuvo información de los instrumentos aplicados, tanto a ellos mismos, como a sus familias, o docentes. Un análisis de la distribución de los estudiantes estudiados en función de diferentes variables aporta la siguiente imagen:

- Según el género de los estudiantes, el 54,8\% de ellos son niños y el 45,2\% son niñas (3071 y 2532, respectivamente).

- Según la edad, el 89\% de los estudiantes tenían 8 ó 9 años; el 3\% menos de esa edad; y el $8 \%$ restante más de esa edad (gráfico 2).

- Según el nivel socioeconómico de las familias, codificado en 11 alternativas (1 menor, 11 mayor), la distribución de los estudiantes se corresponde con una curva Normal, algo platicúrtica (menos apuntada y con colas menos anchas que la normal) y con una ligera asimetría a la izquierda (gráfico 3).

- La media de estudiantes por aula es de 23 estudiantes, con un mínimo de 6 y un máximo de 44. El país donde las aulas cuentan con mayor número de estudiantes es Perú con una media de 33 estudiantes por aula, seguido de Ecuador (26 estudiantes) y Chile (24 estudiantes) (gráfico 4).

- Según la lengua materna, el 92,9\% de los estudiantes tiene el español como lengua materna y el 7,1\% restante otra lengua (5206 y 397 estudiantes, respectivamente).

- Según si han nacido en el país donde estudian o en otro país, el 98,9\% de los estudiantes son nativos del país donde estudian (5541 estudiantes), de los cuales el $4,2 \%$ pertenecen a un grupo étnico minoritario, y un $1,1 \%$ son inmigrantes (61 estudiantes) de los cuales el 3,4\% pertenecen a un grupo étnico minoritario del país.

Gráfico 2. Distribución de la muestra de estudiantes según su edad

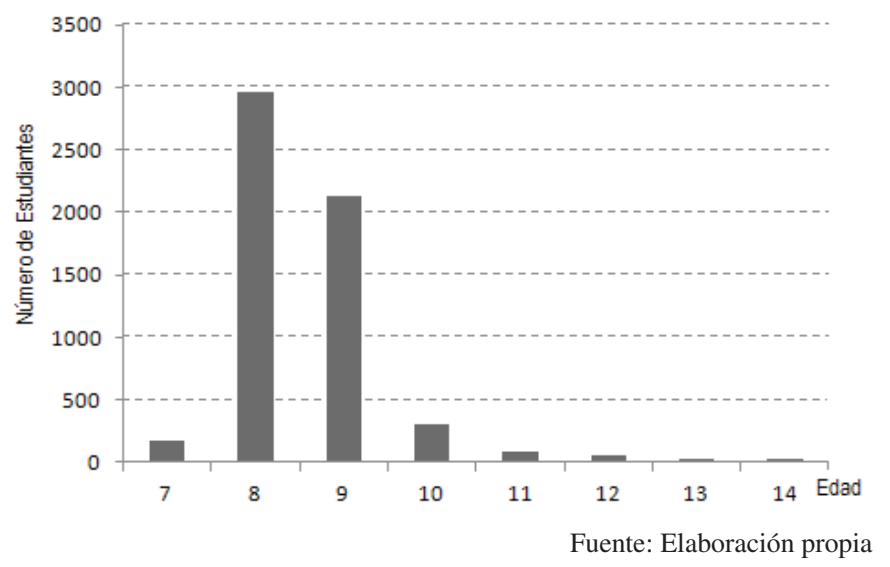


Gráfico 3. Distribución de la muestra de estudiantes según su nivel socioeconómico

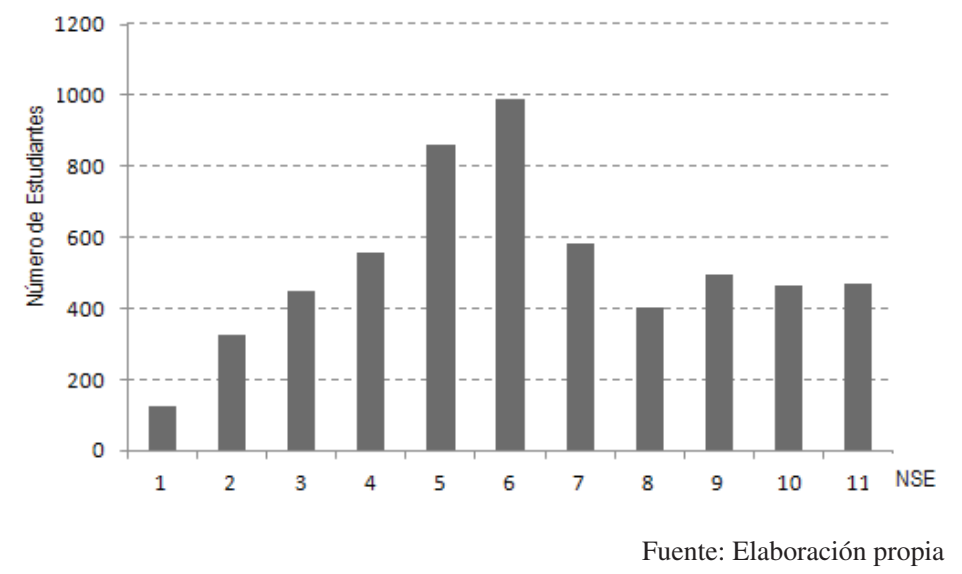

Gráfico 4. Media de estudiantes por aula en función del país

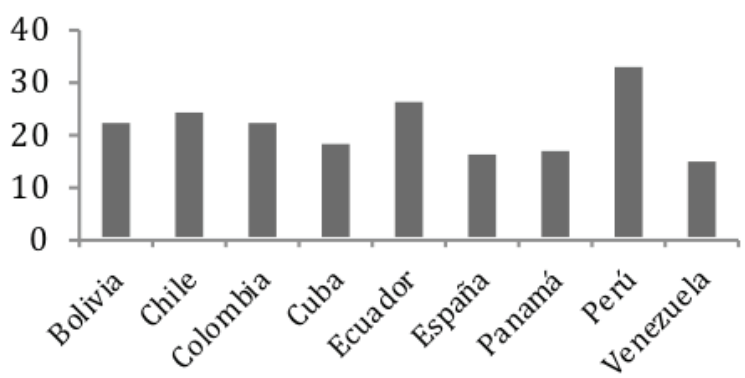

Fuente: Elaboración propia

Se utilizaron cinco tipos de instrumentos:

1. Guía de observación del aula, conformada por tres herramientas. La primera, la utiliza el experto para controlar y medir una serie de variables estables u objetivas (tamaño, luminosidad, limpieza, etc.) cumplimentada antes del transcurso de las sesiones con una escala de cinco categorías, y en términos de Sí/No. La segunda herramienta es una Lista de control para la revisión y contabilización de las actividades en una hora de clase; cumplimentada durante 6 periodos lectivos durante el transcurso de la sesión. Y la tercera herramienta que mide (escala de cinco categorías) una serie de elementos y variables controlados por el investigador a lo largo de una sesión. 
2. Pruebas de Rendimiento en Matemáticas, dos pruebas paralelas, para utilizarlas como medida del rendimiento previo y variable dependiente. Desarrolladas por el Laboratorio Latinoamericano de Evaluación de Calidad de la Educación, de la UNESCO (LLECE 2001). La fiabilidad de las pruebas de Rendimiento en Matemáticas es $\mathrm{a}=0,890$ para la prueba $\mathrm{A}$ (rendimiento previo) y a=0,895 para la prueba $\mathrm{B}$ (rendimiento final). La fiabilidad de las pruebas de Rendimiento en Lengua es a $=0,923$ (prueba A) y a $=0,925$ (prueba B).

3. Test de autoconcepto, elaborado a partir del Self-description Questionnarie (SDQ-I) (Marsh 1988; Marsh, Craven y Debus 1991), está conformado por 56 cuestiones tipo Likert con cuatro alternativas que evalúan el autoconcepto académico (Lectura, Matemáticas y Autoconcepto Escolar General), el Autoconcepto no académico (escala de habilidades físicas, de apariencia física, de relaciones con los pares y de relaciones con los padres), y autoconcepto general. Este Test tiene una fiabilidad de 0,938.

4. Cuestionario sobre la Satisfacción de estudiante con la escuela, compuesto por 19 preguntas aplicado al estudiante sobre su parecer con diferentes elementos de la escuela: sus compañeros, su profesor/a y la escuela en su conjunto. La fiabilidad de este cuestionario es de 0,815 .

5. Cuestionario general del alumnado, consta de 35 cuestiones sencillas en forma de preguntas cerradas, en general con cinco alternativas de respuesta. Entre otras informaciones, se obtiene datos de las variables de ajuste.

6. Cuestionario para las familias, conformado por 41 preguntas cerradas sobre diferentes cuestiones tales como nivel socio-económico y cultural de la familia.

En cada país, los datos fueron recogidos por un equipo de investigación especialista en la temática, desplazándose a cada escuela y permaneciendo en ella no menos de una semana.

\subsection{ANÁLISIS DE DATOS}

Se utilizaron los Modelos Multinivel de cuatro niveles de análisis para determinar la relación entre las diferentes variables de Enseñanza Eficaz y el desarrollo de los estudiantes. El procedimiento de análisis multinivel para cada una de las variables producto fue:

a) Estimar el modelo nulo (Modelo 0), sólo con la variable producto;

b) Calcular el modelo con las variables de ajuste (Modelo 1);

c) Incluir en el modelo ajustado cada una de las variables explicativas de estudio de manera independiente (Modelos 2); y

d) Estimar el modelo final, con todas las variables de ajuste y las explicativas de forma conjunta (Modelo 3).

De esta forma se estimaron tres modelos multinivel (uno para cada variable de producto) con el programa estadístico MLwiN y estimadores de Máxima Verosimilitud, análogos al siguiente:

$y_{i \mathrm{jkl}}=\beta_{0 \mathrm{jkl}}+\beta_{1 \mathrm{jkl}}$ Rdto $_{\text {prev }}+\beta_{\mathrm{ijkl}} \mathrm{NSE}_{\mathrm{ijkl}}+\beta_{3 \mathrm{jkl}} \mathrm{NCult}_{\mathrm{ijkl}}+\beta_{4 \mathrm{jkl}}$ Genero $_{\mathrm{ijkl}}+\beta_{5 \mathrm{jkl}}$ Origen $_{\mathrm{ijkl}}+$ $\beta_{6 \mathrm{kl}}$ Rel_alumnos $_{\mathrm{jkl}}+\beta_{7 \mathrm{kl}}$ Rel_alumnos-prof $_{\mathrm{jkl}}+\beta_{8 \mathrm{kl}}$ Contacto_fis $_{\mathrm{jkl}}+\beta_{9 \mathrm{kl}}$ Comunicacion $_{\mathrm{jkl}}+$ 
$\beta_{10 \mathrm{kl}} \operatorname{Orden}_{\mathrm{jkl}}+\beta_{11 \mathrm{kl}}$ Molestias $_{\mathrm{jkl}}+\beta_{12 \mathrm{kl}}$ Calidez_verbal $_{\mathrm{jkl}}+\beta_{13 \mathrm{kl}}$ Dominio_cont $_{\mathrm{jkl}}+$ $\beta_{14 \mathrm{kl}}$ Planificación ${ }_{\mathrm{jkl}}+\beta_{15 \mathrm{kl}}$ Aseguramiento $_{\mathrm{jkl}}+\beta_{16 \mathrm{kl}}$ Explica_obj $_{\mathrm{jkl}}+\beta_{17 \mathrm{kl}}$ Consolidación $_{-}$ partida $_{\mathrm{jkl}}+\beta_{18 \mathrm{kl}}$ Recordatorio_sistem $_{\mathrm{jkl}}+\beta_{19 \mathrm{kl}}$ Sintesis_cont $_{\mathrm{jkl}}+\beta_{20 \mathrm{kl}}$ Tecnicas_indag $_{\mathrm{jkl}}+$ $\beta_{21 \mathrm{kl}}$ Resol_problemas $_{\mathrm{jkl}}+\beta_{22 \mathrm{kl}}$ Desarrollo_pensam $_{\mathrm{jkl}}+\beta_{23 \mathrm{kl}}$ Ajuste_problemas ${ }_{\mathrm{jkl}}+$ $\beta_{24 \mathrm{kl}}$ Vinculación_cont ${ }_{\mathrm{jkl}}+\beta_{25 \mathrm{kl}}$ Pensamiento_crítico ${ }_{\mathrm{jkl}}+\beta_{26 \mathrm{kl}}$ Colaboración $_{\mathrm{jkl}}+\beta_{27 \mathrm{kl}}$ Buenos $_{3}$ modales $_{\mathrm{jkl}}+\beta_{28 \mathrm{kl}}$ Valores_patrióticos $_{\mathrm{jkl}}+\beta_{29 \mathrm{kl}}$ Refuerzo $_{\mathrm{jkl}}+\beta_{30 \mathrm{kl}} \mathrm{Castigo}_{\mathrm{jkl}}+$ $\beta_{31 \mathrm{kl}}$ Retroalimentación $n_{\mathrm{jkl}}+\beta_{32 \mathrm{kl}}$ Calidad_orientación ${ }_{\mathrm{ijkl}}+\beta_{33 \mathrm{kl}}$ Control_ejecución $_{\mathrm{jkl}}+$ $\beta_{34 \mathrm{kl}}$ Distribución_tiempo $_{\mathrm{jkl}}+\beta_{35 \mathrm{kl}}$ Tiempo_pensar $_{\mathrm{jkl}}+\beta_{36 \mathrm{kl}}$ Puntualidad $_{\mathrm{jkl}}+\varepsilon_{\mathrm{ijkl}}$

$\beta_{0 \mathrm{jkl}}=\beta_{0}+\varphi_{01}+v_{0 \mathrm{kl}}+\mu_{0 \mathrm{jkl}}$

$\beta_{1 \mathrm{jkl}}=\beta_{1}+\varphi_{11}+v_{1 \mathrm{kl}}+\mu_{1 \mathrm{jkl} \ldots} \beta_{5 \mathrm{jkl}}=\beta_{5}+\varphi_{51}+v_{5 \mathrm{kl}}+\mu_{5 \mathrm{jkl}}$

$\beta_{6 \mathrm{kl}}=\beta_{6}+\varphi_{6 \mathrm{l}}+v_{6 \mathrm{kl}} \ldots \beta_{36 \mathrm{kl}}=\beta_{36}+\varphi_{36 \mathrm{l}}+v_{36 \mathrm{kl}}$

Con

$\left[\varepsilon_{0 \mathrm{jikl}}\right] \sim \mathrm{N}\left(0, \Omega_{\varepsilon}\right): \Omega_{\varepsilon}=\left[\sigma^{2}{ }_{\varepsilon 0}\right]$
$\left[\mu_{0 \mathrm{ijk}}\right] \sim \mathrm{N}\left(0, \Omega_{\mu}\right): \Omega_{\varepsilon}=\left[\sigma^{2}{ }^{2}{ }^{\mu}\right]$
$\left[v_{0 \mathrm{ijkl}}\right] \sim \mathrm{N}\left(0, \Omega_{v}\right): \Omega_{v}=\left[\sigma^{2}{ }^{2} 0\right.$
$\left[\varphi_{0 \mathrm{ijkl}}\right] \sim \mathrm{N}\left(0, \Omega_{\varphi}\right): \Omega_{\varphi}=\left[\sigma^{2}{ }_{\varphi 0}\right]$

Donde:

$y_{i j k}$, son las diferentes medidas de desarrollo del estudiante.

Rdto_prev ${ }_{i j k l}$, rendimiento previo del estudiante.

$N S E_{i j k l}$, nivel socio-económico de la familia del estudiante.

NCult $_{i j k l}$, nivel cultural de la familia del estudiante.

Género $_{i j k l}$, si el estudiante es mujer.

Origen $_{i j k l}$, si el estudiante es nativo o inmigrante.

$C l \_A m b_{j k l}$, diferentes variables del clima ambiental del aula.

Prep_sesion ${ }_{j k}$, diferentes variables preparación de las sesiones.

Estruc_sesión ${ }_{j k l}$, diferentes variables de estructuración de las sesiones.

Act_didácticas ${ }_{j k l}$, diferentes variables de actividades didácticas.

Refuerzo_Retroalim ${ }_{j k l}$, diferentes variables de refuerzo y retroalimentación.

Taras_casa $a_{j k l}$, diferentes variables de tareas para casa.

Gestion_tiempo $_{j k l}$, diferentes variables de la gestión del tiempo.

\section{RESULTADOS}

En primer lugar, se presentan los resultados obtenidos para cada una de las tres variables de producto (Rendimiento en Matemáticas, Autoconcepto y Satisfacción con la escuela), para posteriormente ofrecer una panorámica general.

\subsection{FACTORES ASOCIADOS AL RENDIMIENTO EN MATEMÁTICAS}

En el cuadro 3 se presentan los resultados del proceso de modelamiento multinivel para la variable producto rendimiento en Matemáticas. Como muestra el modelo final, una veintena de variables han realizado una aportación significativa, con lo que puede considerarse como factores asociados al logro en Matemáticas (cuadro 4). 
Estudios Pedagógicos XLIV, N $^{\circ}$ 1: 181-205, 2018

FACTORES DE AULA ASOCIADOS AL DESARROLLO INTEGRAL DE LOS ESTUDIANTES: UN ESTUDIO OBSERVACIONAL

Cuadro 4. Resultados de los modelos multinivel para rendimiento en Matemáticas

\begin{tabular}{|c|c|c|c|c|c|c|c|c|}
\hline & \multicolumn{2}{|c|}{ Modelo nulo } & \multicolumn{2}{|c|}{$\begin{array}{c}\text { Modelo con } \\
\text { las v. de ajuste }\end{array}$} & \multicolumn{2}{|c|}{\begin{tabular}{|c|} 
Modelos con las \\
v. individuales
\end{tabular}} & \multicolumn{2}{|c|}{ Modelo final } \\
\hline & Coef. & EE & Coef. & EE & Coef. & $\mathrm{EE}$ & Coef. & $\mathrm{EE}$ \\
\hline \multicolumn{9}{|l|}{ Parte fija } \\
\hline Intercepto & 309,63 & 9,57 & 206,30 & 7,78 & & & 204,06 & 7,12 \\
\hline Rendimiento previo & & & 0,42 & 0,01 & & & 0,42 & 0,01 \\
\hline Nivel socio-económico familia & & & 2,89 & 0,61 & & & 2,93 & 0,62 \\
\hline Nivel cultural familia & & & 3,81 & 0,59 & & & 3,80 & 0,60 \\
\hline Género (Varón-mujer) & & & $-3,37$ & 0,96 & & & $-3,37$ & 0,96 \\
\hline Origen (Nativo-Inmigrante) & & & $-8,04$ & 2,81 & & & $-8,05$ & 2,81 \\
\hline \multicolumn{9}{|l|}{ Clima de aula } \\
\hline Relación entre los alumnos & & & & & $3,06^{*}$ & 2,16 & & \\
\hline $\begin{array}{l}\text { Relación entre alumnos y } \\
\text { profesor }\end{array}$ & & & & & $1,95^{*}$ & 2,18 & & \\
\hline $\begin{array}{l}\text { Contacto físico con los } \\
\text { alumnos positivo }\end{array}$ & & & & & $2,34 *$ & 1,46 & & \\
\hline $\begin{array}{l}\text { Comunicación positiva entre } \\
\text { alumnos }\end{array}$ & & & & & $3,42 *$ & 1,84 & & \\
\hline $\begin{array}{l}\text { Ambiente orientado al } \\
\text { aprendizaje }\end{array}$ & & & & & 4,63 & 1,79 & & \\
\hline $\begin{array}{l}\text { Molestias mutuas entre los } \\
\text { alumnos }\end{array}$ & & & & & $-3,98$ & 1,63 & 2,42 & $-1,11$ \\
\hline $\begin{array}{l}\text { Calidez (verbal) del profesor } \\
\text { hacia los alumnos }\end{array}$ & & & & & $1,72^{*}$ & 1,57 & & \\
\hline \multicolumn{9}{|l|}{ Preparación de las clases } \\
\hline $\begin{array}{l}\text { Dominio del contenido de } \\
\text { enseñanza }\end{array}$ & & & & & 3,84 & 1,90 & & \\
\hline $\begin{array}{l}\text { Planificación de la secuencia } \\
\text { didáctica }\end{array}$ & & & & & 3,81 & 1,69 & & \\
\hline \multicolumn{9}{|l|}{ Estructuración de las sesiones } \\
\hline $\begin{array}{l}\text { Aseguramiento del nivel de } \\
\text { partida }\end{array}$ & & & & & 3,00 & 1,48 & & \\
\hline $\begin{array}{l}\text { Explicación de los objetivos } \\
\text { desde el inicio }\end{array}$ & & & & & 3,13 & 1,31 & & \\
\hline $\begin{array}{l}\text { Consolidación de un punto } \\
\text { antes de avanzar }\end{array}$ & & & & & 3,83 & 1,36 & & \\
\hline $\begin{array}{l}\text { Recordatorio sistemático de los } \\
\text { objetivos }\end{array}$ & & & & & 3,88 & 1,36 & & \\
\hline $\begin{array}{l}\text { Síntesis de los contenidos al } \\
\text { finalizar la sesión }\end{array}$ & & & & & 4,31 & 1,33 & & \\
\hline
\end{tabular}




\begin{tabular}{|c|c|c|c|c|c|c|}
\hline \multicolumn{7}{|l|}{ Actividades didácticas } \\
\hline Técnicas de indagación & & & $2,05^{*}$ & 1,48 & & \\
\hline Resolución de problemas & & & 4,31 & 1,31 & 3,10 & 1,41 \\
\hline $\begin{array}{l}\text { Desarrollo del pensamiento de } \\
\text { los estudiantes }\end{array}$ & & & 4,81 & 1,66 & & \\
\hline $\begin{array}{l}\text { Ajuste de los problemas y } \\
\text { tareas al contenido }\end{array}$ & & & 4,54 & 1,66 & & \\
\hline $\begin{array}{l}\text { Estimulación del pensamiento } \\
\text { crítico y creativo }\end{array}$ & & & 3,72 & 1,42 & & \\
\hline Insistencia en la colaboración & & & 3,45 & 1,30 & & \\
\hline Fomento de buenos modales & & & 4,20 & 1,58 & & \\
\hline \multicolumn{7}{|l|}{$\begin{array}{l}\text { Consolidación de valores } \\
\text { patrióticos y humanistas }\end{array}$} \\
\hline \multicolumn{7}{|l|}{ Refuerzo y retroalimentación } \\
\hline Refuerzo & & & 2,90 & 1,44 & & \\
\hline Castigo & & & $-2,32 *$ & 1,96 & & \\
\hline $\begin{array}{l}\text { Retroalimentación y } \\
\text { corrección informativas }\end{array}$ & & & 3,98 & 1,32 & & \\
\hline \multicolumn{7}{|l|}{ Tareas para casa } \\
\hline Calidad de su orientación & & & 3,92 & 1,56 & & \\
\hline Control de la ejecución & & & 2,64 & 1,30 & & \\
\hline \multicolumn{7}{|l|}{ Gestión del tiempo } \\
\hline $\begin{array}{l}\text { Distribución del tiempo en la } \\
\text { clase }\end{array}$ & & & 4,88 & 1,67 & 3,31 & 1,27 \\
\hline $\begin{array}{l}\text { Concesión de tiempo para que } \\
\text { los alumnos piensen }\end{array}$ & & & $2,63 *$ & 1,54 & & \\
\hline Puntualidad de los alumnos & & & $2,10 *$ & 1,89 & & \\
\hline \multicolumn{7}{|l|}{$\begin{array}{l}\text { Coeficiente de Correlacion } \\
\text { Intraclase }(\mathrm{CCI})\end{array}$} \\
\hline País & 0,2723 & 0,2051 & & & 0,1783 & \\
\hline Escuela & 0,1412 & 0,0549 & & & 0,0421 & \\
\hline Aula & 0,1286 & 0,1665 & & & 0,1318 & \\
\hline Estudiante & 0,4578 & 0,5735 & & & 0,6478 & \\
\hline \multicolumn{7}{|l|}{ Componentes de la varianza } \\
\hline País & $27,23 \%$ & $20,51 \%$ & & & $17,83 \%$ & \\
\hline Escuela & $14,12 \%$ & $5,49 \%$ & & & $4,21 \%$ & \\
\hline Aula & $12,86 \%$ & $16,65 \%$ & & & $13,18 \%$ & \\
\hline Estudiante & $45,78 \%$ & $57,35 \%$ & & & $64,78 \%$ & \\
\hline Explicado & & $32,91 \%$ & & & $40,61 \%$ & \\
\hline
\end{tabular}


Llama la atención del modelo final que sólo aparezcan tres variables de proceso: una de clima (los estudiantes se molestan entre ellos), otra de actividades didácticas (la realización de problemas) y una tercera relacionada con la gestión del tiempo (la distribución del tiempo de las clases). Seguramente se deba a que todas las variables de proceso están relacionadas, conformando una "cultura de eficacia". Ello unido al débil efecto de todas las variables, hace que el efecto de esas variables se compense.

\subsection{FACTORES ASOCIADOS AL AUTOCONCEPTO}

La segunda variable de producto analizada es el autoconcepto. El proceso de modelamiento multinivel ofrece una imagen completamente diferente al obtenido para Matemáticas. Efectivamente, en primer lugar, son diferentes las variables de ajuste que realizan una aportación significativa: Nivel socio económico y nivel cultural de las familias, y origen (inmigrante y nativo). El ser niño o niña no parece afectar al autoconcepto de los estudiantes (cuadro 5).

Mucho más interesante para los propósitos de esta investigación es, sin embargo, el estudio de los modelos en los que se han incorporado las variables de proceso de forma individual. En este caso, sólo cuatro variables han realizado una aportación significativa: Relación entre los alumnos, Consolidación de valores patrióticos y humanistas, Puntualidad de los alumnos y Concesión de tiempo para que los alumnos piensen. De estas cuatro, sólo una aparece en el modelo final, la concesión de tiempo para que los alumnos piensen.

Los datos indican que cuatro elementos de aula favorecen el autoconcepto de los estudiantes: i) la relación que tienen entre sí los alumnos tiene efectos directamente positivos con el autoconcepto; ii) si el docente deja tiempo para que los estudiantes piensen, mayor será el autoconcepto de estos; iii) cuanto mayor es la puntualidad de los estudiantes, mayor su autoconcepto, y iv) el esfuerzo del docente por consolidar los valores patrióticos tiene un efecto positivo sobre el autoconcepto de los estudiantes.

Cuadro 5. Resultados de los modelos multinivel para Autoconcepto

\begin{tabular}{|l|c|c|c|c|c|c|c|c|c|}
\hline & \multicolumn{2}{|c|}{$\begin{array}{c}\text { Modelo } \\
\text { nulo }\end{array}$} & \multicolumn{2}{c|}{$\begin{array}{c}\text { Modelo con } \\
\text { las v de ajuste }\end{array}$} & $\begin{array}{c}\text { Modelos con las } \\
\text { v. individuales }\end{array}$ & \multicolumn{2}{|c|}{$\begin{array}{c}\text { Modelo } \\
\text { final }\end{array}$} \\
\hline & Coef. & EE & Coef. & EE & Coef. & EE & Coef. & EE \\
\hline Parte fija & & & & & & & & \\
\hline Intercepto & 251,51 & 6,27 & 252,04 & 5,80 & & & 249,50 & 5,68 \\
\hline Nivel socio-económico familia & & & 1,85 & 0,81 & & & 1,89 & 0,81 \\
\hline Nivel cultural familia & & & 2,62 & 0,77 & & & 2,57 & 0,80 \\
\hline Nativo-Inmigrante & & & $-8,62$ & 3,70 & & & $-8,62$ & 3,70 \\
\hline Clima de aula & & & & & & & & \\
\hline Relación entre los alumnos & & & & & 4,27 & 2,02 & & \\
\hline Relación entre alumnos y profesor & & & & & $3,54 *$ & 2,04 & & \\
\hline $\begin{array}{l}\text { Contacto físico con los alumnos } \\
\text { positivo }\end{array}$ & & & & & $1,70^{*}$ & 1,37 & & \\
\hline
\end{tabular}




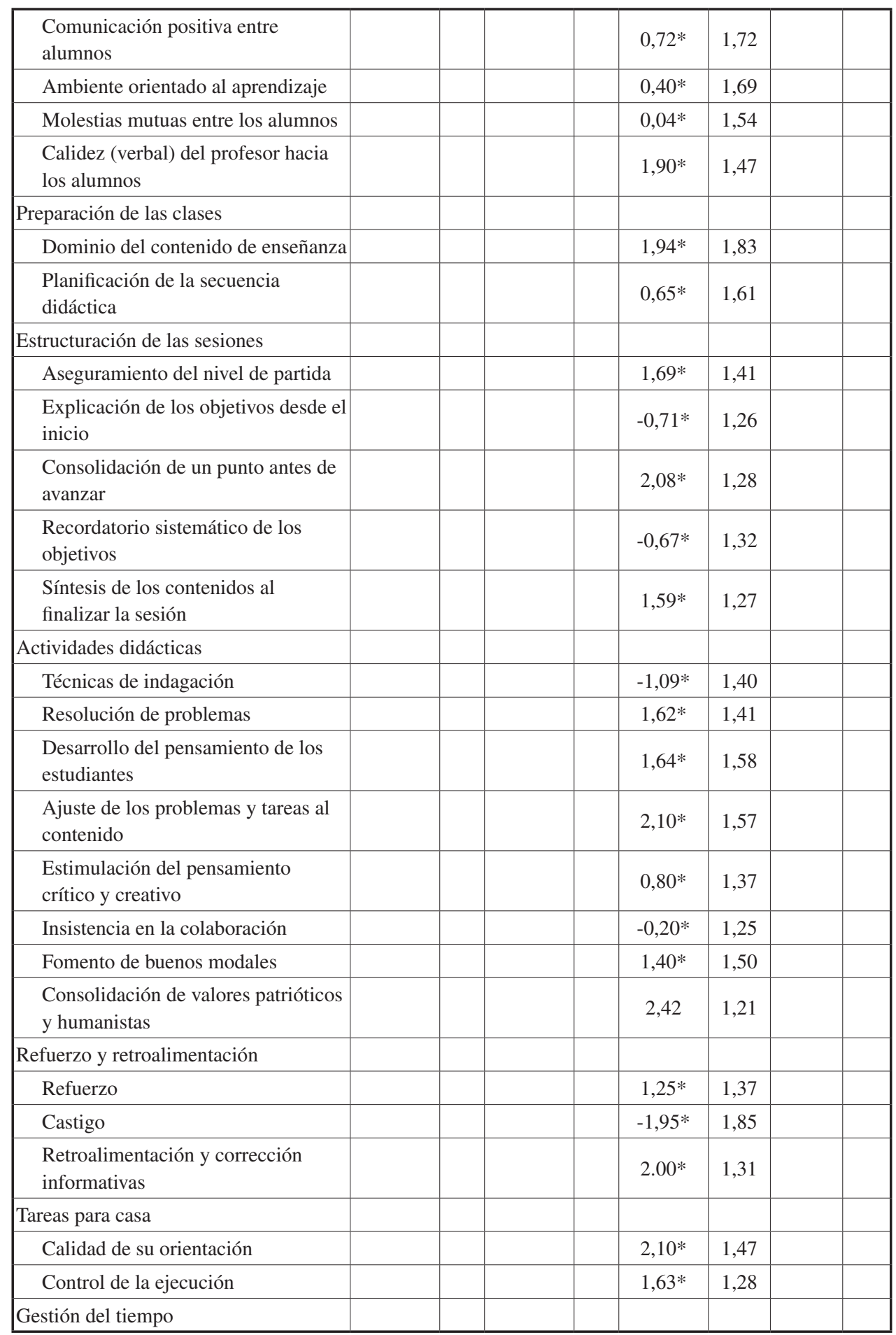




\begin{tabular}{|c|c|c|c|c|c|c|}
\hline Distribución del tiempo en la clase & & & $-1,49 *$ & 1,60 & & \\
\hline $\begin{array}{l}\text { Concesión de tiempo para que los } \\
\text { alumnos piensen }\end{array}$ & & & 3,79 & 1,43 & 3,79 & 1,43 \\
\hline Puntualidad de los alumnos & & & 3,41 & 1,70 & & \\
\hline \multicolumn{7}{|c|}{ Coeficiente de Correlación Intraclase (CCI) } \\
\hline País & 0,1201 & 0,1044 & & & 0,0986 & \\
\hline Escuela & 0,0648 & 0,0586 & & & 0,0530 & \\
\hline Aula & 0,0825 & 0,0832 & & & 0,0842 & \\
\hline Estudiante & 0,7327 & 0,7538 & & & 0,7641 & \\
\hline \multicolumn{7}{|l|}{ Componentes de la varianza } \\
\hline País & $12,01 \%$ & $10,44 \%$ & & & $9,86 \%$ & \\
\hline Escuela & $6,48 \%$ & $5,86 \%$ & & & $5,30 \%$ & \\
\hline Aula & $8,25 \%$ & $8,32 \%$ & & & $8,42 \%$ & \\
\hline Estudiante & $73,27 \% \%$ & $75,38 \%$ & & & $76,41 \%$ & \\
\hline Explicado & & $3,16 \%$ & & & $4,75 \%$ & \\
\hline
\end{tabular}

Nota: * Variables no significativas $(\alpha=0,05)$. Fuente: Elaboración propia.

\subsection{FACTORES ASOCIADOS A LA SATISFACCIÓN DEL ESTUDIANTE CON LA ESCUELA}

La tercera y última variable de producto estudiada, directamente relacionada con el bienestar del alumno, es su satisfacción con la escuela. Los resultados del proceso de modelado multinivel (cuadro 6) muestran, en primer lugar, las variables de ajuste cuyos coeficientes han hecho una aportación significativa: son las variables de entrada asociadas a la satisfacción de los estudiantes. Y, aunque queda fuera de los objetivos de este trabajo, es interesante señalar que:

- Los estudiantes cuyos padres tiene un mayor nivel socioeconómico están más satisfechos con la escuela que los de nivel bajo.

- Los alumnos que no han nacido en el país donde estudian están más descontentos con la escuela que los que han nacido en el país; los estudiantes cuya lengua materna no es el español (normalmente pertenecientes a grupos indígenas) están más descontentos con la escuela.

- Las estudiantes mujeres están más contentas con la escuela que sus compañeros hombres.

- Sin embargo, el nivel cultural de las familias, según estos datos, parece no influir.

Ello nos da la imagen de una escuela dirigida a estudiantes de nivel socioeconómico acomodado, nativos y de grupo cultural minoritarios.

De los modelos con las variables individuales se obtienen los factores de aula asociados con la satisfacción de los estudiantes. En este caso, una gran cantidad de variables definen los grandes factores de aula: Clima de aula, Estructuración de las Sesiones, Actividades Didácticas, Refuerzo y Retroalimentación, Tareas para casa y Gestión del tiempo. De entre 
todas las variables son sólo tres las que aparecen en el modelo final: la relación entre los alumnos, el desarrollo del pensamiento de los estudiantes y la calidad de su orientación en las tareas para casa. De nuevo la existencia de tan sólo tres variables evidencia la relación de dependencia entre unas y otras variables de proceso. Los resultados del modelo final confirman que en la medida que exista un clima de confianza, complicidad y de trabajo entre los estudiantes, se apueste hacia el aprendizaje de todos los estudiantes y se afiancen los aprendizajes a través de ajustadas tareas para casa los estudiantes sentirán mayor satisfacción hacia la escuela.

Cuadro 6. Resultados de los modelos multinivel para Satisfacción del estudiante con la escuela

\begin{tabular}{|c|c|c|c|c|c|c|c|c|}
\hline & \multicolumn{2}{|c|}{$\begin{array}{l}\text { Modelo } \\
\text { nulo }\end{array}$} & \multicolumn{2}{|c|}{$\begin{array}{c}\text { Modelo con las } \\
\mathrm{v} \text { de ajuste }\end{array}$} & \multicolumn{2}{|c|}{$\begin{array}{c}\text { Modelos con las } \\
\text { v. individuales }\end{array}$} & \multicolumn{2}{|c|}{$\begin{array}{l}\text { Modelo } \\
\text { final }\end{array}$} \\
\hline & Coef. & $\mathrm{EE}$ & Coef. & $\mathrm{EE}$ & Coef. & $\mathrm{EE}$ & Coef. & $\mathrm{EE}$ \\
\hline \multicolumn{9}{|l|}{ Parte fija } \\
\hline Intercepto & 251,01 & 3,38 & 241,20 & 4,11 & & & 233,43 & 4,04 \\
\hline Nivel socio-económico familia & & & 3,27 & 0,82 & & & 3,03 & 0,81 \\
\hline Género (Varón-mujer) & & & 4,40 & 1,39 & & & 4,84 & 1,35 \\
\hline Nativo-inmigrante & & & $-10,25$ & 3,90 & & & $-10,52$ & 3,89 \\
\hline Lengua Materna (Español-Otra) & & & 9,00 & 3,18 & & & 9,90 & 3,13 \\
\hline \multicolumn{9}{|l|}{ Clima de aula } \\
\hline Relación entre los alumnos & & & & & 5,73 & 1,87 & 4,26 & 1,88 \\
\hline Relación entre alumnos y profesor & & & & & 6,11 & 1,87 & & \\
\hline $\begin{array}{l}\text { Contacto físico con los alumnos } \\
\text { positivo }\end{array}$ & & & & & 3,29 & 1,26 & & \\
\hline $\begin{array}{l}\text { Comunicación positiva entre los } \\
\text { alumnos }\end{array}$ & & & & & 4,35 & 1,61 & & \\
\hline Ambiente orientado al aprendizaje & & & & & 3,50 & 1,56 & & \\
\hline Molestias mutuas entre los alumnos & & & & & $-3,06$ & 1,36 & & \\
\hline $\begin{array}{l}\text { Calidez (verbal) del profesor hacia } \\
\text { los alumnos }\end{array}$ & & & & & 3,75 & 1,36 & & \\
\hline \multicolumn{9}{|l|}{ Preparación de las clases } \\
\hline $\begin{array}{l}\text { Dominio del contenido de } \\
\text { enseñanza }\end{array}$ & & & & & $3,01 *$ & 1,70 & & \\
\hline $\begin{array}{l}\text { Planificación de la secuencia } \\
\text { didáctica }\end{array}$ & & & & & $1,74 *$ & 1,45 & & \\
\hline \multicolumn{9}{|l|}{ Estructuración de las sesiones } \\
\hline Aseguramiento del nivel de partida & & & & & $1,68 *$ & 1,26 & & \\
\hline $\begin{array}{l}\text { Explicación de los objetivos desde } \\
\text { el inicio }\end{array}$ & & & & & $1,27 *$ & 1,11 & & \\
\hline $\begin{array}{l}\text { Consolidación de un punto antes de } \\
\text { avanzar }\end{array}$ & & & & & $1,24 *$ & 1,17 & & \\
\hline
\end{tabular}




\begin{tabular}{|c|c|c|c|c|c|c|}
\hline $\begin{array}{l}\text { Recordatorio sistemático de los } \\
\text { objetivos }\end{array}$ & & & 2,38 & 1,18 & & \\
\hline $\begin{array}{l}\text { Síntesis de los contenidos al } \\
\text { finalizar la sesión }\end{array}$ & & & 2,37 & 1,17 & & \\
\hline \multicolumn{7}{|l|}{ Actividades didácticas } \\
\hline Técnicas de indagación & & & $1,60 *$ & 1,27 & & \\
\hline Resolución de problemas & & & $1,62 *$ & 1,29 & & \\
\hline $\begin{array}{l}\text { Desarrollo del pensamiento de los } \\
\text { estudiantes }\end{array}$ & & & 5,02 & 1,41 & 3,21 & 1,53 \\
\hline $\begin{array}{l}\text { Ajuste de los problemas y tareas al } \\
\text { contenido }\end{array}$ & & & 3,00 & 1,43 & & \\
\hline $\begin{array}{l}\text { Vinculación del contenido de } \\
\text { enseñanza con la vida }\end{array}$ & & & 2,54 & 1,27 & & \\
\hline $\begin{array}{l}\text { Estimulación del pensamiento } \\
\text { crítico y creativo }\end{array}$ & & & $1,23 *$ & 1,22 & & \\
\hline Insistencia en la colaboración & & & $0,92 *$ & 1,34 & & \\
\hline Fomento de buenos modales & & & 4,03 & 1,37 & & \\
\hline $\begin{array}{l}\text { Consolidación de valores } \\
\text { patrióticos y humanistas }\end{array}$ & & & 3,17 & 1,08 & & \\
\hline \multicolumn{7}{|l|}{ Refuerzo y retroalimentación } \\
\hline Refuerzo & & & 3,51 & 1,25 & & \\
\hline Castigo & & & $-2,37 *$ & 1,69 & & \\
\hline $\begin{array}{l}\text { Retroalimentación y corrección } \\
\text { informativas }\end{array}$ & & & 3,83 & 1,22 & & \\
\hline \multicolumn{7}{|l|}{ Tareas para casa } \\
\hline Calidad de su orientación & & & 4,55 & 1,36 & 2,06 & 1,44 \\
\hline Control de la ejecución & & & $2,22 *$ & 1,34 & & \\
\hline \multicolumn{7}{|l|}{ Gestión del tiempo } \\
\hline Distribución del tiempo en la clase & & & 3,88 & 1,46 & & \\
\hline $\begin{array}{l}\text { Concesión de tiempo para que los } \\
\text { alumnos piensen }\end{array}$ & & & 3,42 & 1,34 & & \\
\hline Puntualidad de los alumnos & & & 5,19 & 1,60 & & \\
\hline \multicolumn{7}{|l|}{$\begin{array}{l}\text { Coeficiente de Correlación Intraclase } \\
\text { (CCI) }\end{array}$} \\
\hline País & 0,0287 & 0,0189 & & 0,0088 & & \\
\hline Escuela & 0,0405 & 0,0179 & & 0,0064 & & \\
\hline Aula & 0,0799 & 0,0829 & & 0,0825 & & \\
\hline Estudiante & 0,8510 & 0,8804 & & 0,9024 & & \\
\hline \multicolumn{7}{|l|}{ Componentes de la varianza } \\
\hline País & $2,87 \%$ & $1,89 \%$ & & $0,88 \%$ & & \\
\hline Escuela & $4,05 \%$ & $1,79 \%$ & & $0,64 \%$ & & \\
\hline Aula & $7,99 \%$ & $8,29 \%$ & & $8,25 \%$ & & \\
\hline Estudiante & $85,10 \%$ & $88,04 \%$ & & $90,24 \%$ & & \\
\hline Explicado & & $3,65 \%$ & & $5,95 \%$ & & \\
\hline
\end{tabular}

Nota: * Variables no significativas $(\alpha=0,05)$. Fuente: Elaboración propia. 


\subsection{PANORÁMICA GENERAL}

Una mirada conjunta de las aportaciones de las diferentes variables ofrece una imagen sólida, coherente y llena de matices acerca de los factores de aula que indicen en el desarrollo de los estudiantes. Así, es posible afirmar que los siete factores estudiados inciden, de una forma u otra, en el desarrollo de los estudiantes (cuadro 7).

Cuadro 7. Resumen de las aportaciones de las variables en los diferentes modelos

\begin{tabular}{|c|c|c|c|c|}
\hline & & $\begin{array}{l}\text { Rend. } \\
\text { Matemáticas }\end{array}$ & Autoconcepto & $\begin{array}{l}\text { Satisfacción } \\
\text { con la escuela }\end{array}$ \\
\hline \multirow{7}{*}{ Clima de aula } & Relación entre los alumnos & & $\mathrm{X}$ & $\mathrm{XX}$ \\
\hline & $\begin{array}{l}\text { Relación entre alumnos y } \\
\text { profesor }\end{array}$ & & & $\mathrm{X}$ \\
\hline & $\begin{array}{l}\text { Contacto físico con los alumnos } \\
\text { positivo }\end{array}$ & & & $\mathrm{X}$ \\
\hline & $\begin{array}{l}\text { Comunicación positiva entre } \\
\text { alumnos }\end{array}$ & & & $\mathrm{X}$ \\
\hline & $\begin{array}{l}\text { Ambiente orientado al } \\
\text { aprendizaje }\end{array}$ & $\mathrm{X}$ & & $\mathrm{X}$ \\
\hline & $\begin{array}{l}\text { Molestias mutuas entre los } \\
\text { alumnos }\end{array}$ & $\mathrm{XX}$ & & $\mathrm{X}$ \\
\hline & $\begin{array}{l}\text { Calidez (verbal) del profesor } \\
\text { hacia los alumnos }\end{array}$ & & & $\mathrm{X}$ \\
\hline \multirow{2}{*}{$\begin{array}{l}\text { Preparación de } \\
\text { las clases }\end{array}$} & $\begin{array}{l}\text { Dominio del contenido de } \\
\text { enseñanza }\end{array}$ & $\mathrm{X}$ & & \\
\hline & $\begin{array}{l}\text { Planificación de la secuencia } \\
\text { didáctica }\end{array}$ & $\mathrm{X}$ & & \\
\hline \multirow{5}{*}{$\begin{array}{l}\text { Estructuración de } \\
\text { las sesiones }\end{array}$} & $\begin{array}{l}\text { Aseguramiento del nivel de } \\
\text { partida }\end{array}$ & $\mathrm{X}$ & & \\
\hline & $\begin{array}{l}\text { Explicación de los objetivos } \\
\text { desde el inicio }\end{array}$ & $\mathrm{X}$ & & \\
\hline & $\begin{array}{l}\text { Consolidación de un punto antes } \\
\text { de avanzar }\end{array}$ & $\mathrm{X}$ & & \\
\hline & $\begin{array}{l}\text { Recordatorio sistemático de los } \\
\text { objetivos }\end{array}$ & $\mathrm{X}$ & & $\mathrm{X}$ \\
\hline & $\begin{array}{l}\text { Síntesis de los contenidos al } \\
\text { finalizar la sesión }\end{array}$ & $X$ & & $X$ \\
\hline
\end{tabular}




\begin{tabular}{|c|c|c|c|c|}
\hline \multirow{9}{*}{$\begin{array}{l}\text { Actividades } \\
\text { didácticas }\end{array}$} & Técnicas de indagación & & & \\
\hline & Resolución de problemas & $\mathrm{XX}$ & & \\
\hline & $\begin{array}{l}\text { Desarrollo del pensamiento de } \\
\text { los estudiantes }\end{array}$ & $X$ & & $\mathrm{XX}$ \\
\hline & $\begin{array}{l}\text { Ajuste de los problemas y tareas } \\
\text { al contenido }\end{array}$ & $\mathrm{X}$ & & $X$ \\
\hline & $\begin{array}{l}\text { Vinculación del contenido de } \\
\text { enseñanza con la vida }\end{array}$ & & & $\mathrm{X}$ \\
\hline & $\begin{array}{l}\text { Estimulación del pensamiento } \\
\text { crítico y creativo }\end{array}$ & $X$ & & \\
\hline & Insistencia en la colaboración & $\mathrm{X}$ & & \\
\hline & Fomento de buenos modales & $\mathrm{X}$ & & $\mathrm{X}$ \\
\hline & $\begin{array}{l}\text { Consolidación de valores } \\
\text { patrióticos y humanistas }\end{array}$ & & $\mathrm{X}$ & $\mathrm{X}$ \\
\hline \multirow{3}{*}{$\begin{array}{l}\text { Refuerzo y } \\
\text { retroalimentación }\end{array}$} & Refuerzo & $X$ & & $\mathrm{X}$ \\
\hline & Castigo & & & \\
\hline & $\begin{array}{l}\text { Retroalimentación y corrección } \\
\text { informativas }\end{array}$ & $X$ & & $\mathrm{X}$ \\
\hline \multirow{2}{*}{ Tareas para casa } & $\begin{array}{l}\text { Calidad de su orientación en las } \\
\text { tareas para casa }\end{array}$ & $X$ & & XX \\
\hline & $\begin{array}{l}\text { Control de la ejecución de las } \\
\text { tareas para casa }\end{array}$ & $X$ & & \\
\hline \multirow{3}{*}{$\begin{array}{l}\text { Gestión del } \\
\text { tiempo }\end{array}$} & Puntualidad de los alumnos & & & $X$ \\
\hline & $\begin{array}{l}\text { Concesión de tiempo para que los } \\
\text { alumnos piensen }\end{array}$ & & XX & $\mathrm{X}$ \\
\hline & $\begin{array}{l}\text { Distribución del tiempo en la } \\
\text { clase }\end{array}$ & $\mathrm{XX}$ & & $\mathrm{X}$ \\
\hline
\end{tabular}

Notas: (X): Aportación significativa en los modelos con las variables individuales. (XX): Aportación significativa en los modelos finales. Fuente: Elaboración propia.

El clima del aula incide tanto en el rendimiento en matemáticas del estudiante, en su autoconcepto como en su satisfacción con la escuela; pero de una forma diferenciada. Efectivamente, los coeficientes de las siete variables que componen el factor Satisfacción con la escuela realizan una aportación significativa al modelo ajustado. De tal forma que no sería arriesgado afirmar que los estudiantes están contentos con la escuela en función de su relación con sus compañeros y docentes. Sin embargo, sólo dos variables están relacionadas con el rendimiento en Matemáticas: el Ambiente de aula orientado al aprendizaje, y la 
existencia de molestias mutuas entre el alumnado, la primera una incidencia positiva y la segunda negativa. El autoconcepto del estudiante, por último, solo parece estar relacionado con el indicador "Relación entre los alumnos".

La preparación de las clases por parte del docente tiene una incidencia determinante en el aprendizaje académico de los estudiantes. Los datos son concluyentes en afirmar que los mejores docentes son aquellos que le dedican tiempo antes de las clases. Sin embargo, ese factor no está asociado ni al autoconcepto ni a la satisfacción del alumno en la escuela.

El factor denominado estructuración de las clases y conformado por variables tales como Aseguramiento del nivel de partida, Explicación de los objetivos desde el inicio, Consolidación de un punto antes de avanzar, Recordatorio sistemático de los objetivos, y Síntesis de los contenidos al finalizar la sesión, tienen una incidencia significativa sobre el rendimiento de los estudiantes en Matemáticas. Sin embargo, no parece tener incidencia en el autoconcepto del estudiante y sólo las dos últimas variables, en su satisfacción.

Las actividades didácticas tienen una incidencia significativa sobre el rendimiento académico de los estudiantes y su satisfacción con la escuela. De todos los indicadores que conforman este gran factor, la consolidación de valores patrióticos y humanistas, es el único el que parece afectar al Autoconcepto del estudiante.

El factor Evaluación y retroalimentación desvela la importancia del refuerzo, la retroalimentación y corrección informativa sobre el desarrollo académico del estudiante y su satisfacción con la escuela. Así mismo, se confirma la irrelevancia del castigo y de nuevo, el autoconcepto parece no resultar influido por los indicadores seleccionados.

Las tareas para casa se consolidan como un factor con alta incidencia sobre el rendimiento académico de los estudiantes y, en concreto cómo se orienten y ajusten hacia cada estudiante incide además en los niveles de satisfacción con la escuela. Estos indicadores no parecen influir sobre el autoconcepto.

Por último, los datos hablan de la importancia de la gestión del tiempo del aula por parte de los docentes para lograr el desarrollo de los estudiantes. El adecuado uso de las tres variables estudiadas - puntualidad de los estudiantes, concesión de tiempo para que los estudiantes aprendan y distribución del tiempo en la clase-, supone elementos clave que garantizan la eficacia del docente.

\section{DISCUSIÓN}

El estudio realizado ratifica la importancia de los factores de aula para el completo desarrollo de los estudiantes. El diseño de la presente investigación y el abordaje del mismo a partir de la observación sistemática por parte de expertos educativos y el trabajo no sólo con medidas de rendimiento cognitivo, también socio-afectivo es uno de los elementos más enriquecedores del estudio. Se esquivan así las principales limitaciones encontradas en anteriores estudios como el uso de cuestionarios que recogen autopercepciones del docente y/o del estudiante, y la consideración de que es el rendimiento académico el único indicador de la eficacia de la enseñanza.

Los resultados encontrados demuestran la existencia de siete factores que inciden de manera significativa sobre el desarrollo de académico y socio-afectivo de los estudiantes: Clima de aula, Preparación de las clases, Lecciones estructuradas, Actividades didácticas, Evaluación y retroalimentación, Tareas para casa, y Gestión del tiempo. 
De acuerdo con la revisión de Thapa et al., los resultados de este estudio confirman que, entre otros, el clima del aula está asociado con el rendimiento del estudiante (Thapa et al. 2013). Los datos obtenidos en la presente investigación indican que la presencia de comportamientos disruptores entre los estudiantes reduce en -2,42 puntos el rendimiento en matemáticas, que la existencia de buenas relaciones entre los estudiantes aumenta en 4,3 puntos la satisfacción del estudiante con su escuela.

En coherencia con los resultados obtenidos por Gran, Hindman y Stronge que señalaron que el 52\% del éxito de los estudiantes en la asignatura de Matemáticas dependía de cómo el docente preparara sus clases (Gran, Hindman y Stronge 2010). Los datos obtenidos reflejan que en la medida que el docente domine los contenidos de enseñanza y planifique la secuencia didáctica de la sesión sus estudiantes mejorarán sus resultados en Matemáticas, casi 4 puntos.

Tal y como señalan Killen y Hunt, Wiseman y Touzel los docentes que estructuran sus clases hacia la consecución de objetivos de carácter instructivo y persiguen el desarrollo de destrezas de carácter superior son docentes cuyos estudiantes obtienen mejores resultados (Killen 2006; Hunt, Wiseman y Touzel 2009). A este respecto, los datos reflejan que en la medida que el docente recuerde de manera sistemática los objetivos de la sesión y sintetice los contenidos trabajados al finalizar la clase los estudiantes aumentarán 2,3 puntos y 2,4 puntos respectivamente su rendimiento en Matemáticas.

Así mismo, las actividades didácticas son ejes principales del rendimiento académico y la satisfacción del estudiante con la escuela. Los resultados obtenidos son coherentes con los encontrados por Hunt, Wiseman, y Touzel, los datos reflejan que por cada desviación típica que aumente el uso de la resolución de problemas por parte del docente, los estudiantes aumentarán 3,1 puntos su rendimiento en Matemáticas. Así mismo, por cada desviación típica que aumente el uso de actividades que desarrollen el pensamiento de los estudiantes, éstos verán aumentado en 3,2 puntos sus niveles de satisfacción con la escuela. De lo cual se puede concluir que, así como sugirió la investigación realizada por Killen el tipo de actividades que se desarrollen dentro del aula afecta de manera significativa al desarrollo cognitivo y socioafectivo de los estudiantes (Hunt, Wiseman y Touzel 2009; Killen 2006).

Los beneficios que conlleva la evaluación y la retroalimentación de los aprendizajes a los estudiantes están sobradamente contrastados (Brookhart 2009; Orlich et al. 2010). En efecto, los resultados obtenidos muestran los efectos del refuerzo y la retroalimentación sobre el rendimiento en Matemáticas y en la satisfacción de los estudiantes con el centro: 3,4 y 3,6 puntos respectivamente.

Las tareas para casa, instigadoras de más de una disputa entre madres, docentes y estudiantes, se consolida tal y como señalaron Trautwein y Köller como elementos claves para aumentar el rendimiento de los estudiantes, en concreto en 3,3 puntos el rendimiento en Matemáticas y en 2 puntos su nivel de satisfacción con la escuela (Trautwein y Köller 2003).

El tiempo es quizá una de las variables que más ha preocupado a los investigadores en educación (Murillo 2007a). Los resultados de la presente investigación señalan que cuanto mejor se distribuya el tiempo en la clase, mejor será el rendimiento que obtendrán los estudiantes en Matemáticas (en concreto 3,3 puntos más) y, cuanto más tiempo se destine para que los alumnos piensen, el autoconcepto de éstos también se verá incrementado (casi 4 puntos más). 
Por último, el estudio ha explicado mucha varianza de rendimiento y poca de autoconcepto y satisfacción del estudiante hacia la escuela, esto es razonable sabiendo que el rendimiento tiene mucho peso la escuela y en las otras, por lo que se sabe (Murillo 2007b) es muy bajo

\section{CONCLUSIONES}

La investigación sobre la Enseñanza Eficaz ha aportado y continúa aportando importantes hallazgos para lograr ofrecer en las aulas una enseñanza de calidad. El apoyo que estos estudios dan a los sistemas educativos actuales contribuye a reconducir las aulas, y con ello las escuelas, hacia la igualdad de oportunidades, maximizar el tiempo en ambientes educativos, ofrecer un amplio abanico de actividades educativas y cuidar los ambientes de estudio. Sin duda alguna, la verificación de los factores de Enseñanza Eficaz validados en este estudio supone un aporte a tener en cuenta para futuras investigaciones que busquen confirmar factores de enseñanza eficaz. Y no sólo en el aprendizaje de Matemáticas o Lengua, también en Autoconcepto y Satisfacción, elementos tan importantes como olvidados.

Los resultados obtenidos de este estudio tienen una clara aplicación práctica: desde contribuir a la mejora de la formación de profesores, pasando por el diseño de sistemas de evaluación del desempeño del docente, o aportar datos para la auto-observación y la reflexión de profesores y profesoras que desemboquen en una optimización de su trabajo.

Desde el punto de vista de la metodología, cuatro elementos caracterizan la presente investigación y que le hacen ser muy destacable. En primer lugar, obtiene información mediante la observación directa por parte de investigadores experimentados de 248 aulas de características pretendidamente diferentes utilizando un mismo protocolo estandarizado. De esta forma, se avanza en una de las principales limitaciones de este tipo de estudios, no es el docente que dice lo que hace ni la opinión de los estudiantes, es lo que el docente hace y el investigador observa. En segundo lugar, utiliza tres variables de producto complementarias: Rendimiento en Matemáticas, Autoconcepto y Satisfacción con la escuela, de tal forma que se supera la tradicional visión de los estudios de eficacia centrados sólo en medidas de producto cognitivo. También destaca el utilizar Modelos Multinivel de 4 niveles, algo extremadamente infrecuente y el ser un estudio internacional, desarrollado en colaboración por nueve centros de investigación de otros tantos países.

Sin embargo, o quizá gracias a ello, este trabajo muestra lo mucho que queda por avanzar en esta línea de trabajo. Desde replicarlo no solo para Educación Secundaria, también para estudios posobligatorios y para Educación Infantil. Precisamente la metodología observacional resulta de especial interés en Educación Infantil, tanto para estudiar el comportamiento de los y las docentes como para medir las variables de producto tanto cognitivo como afectivo o psicomotriz.

La investigación y la experiencia nos dicen que el factor que más incide en el desarrollo (al menos de desarrollo cognitivo) de los niños y niñas es lo que hacen los docentes en el aula. Si y sólo si queremos una mejor educación que nos lleve a una sociedad más junta e inclusiva, necesitamos conocer más y mejor como son las aulas que consiguen ese desarrollo integral. Este artículo solo busca eso. 


\section{REFERENCIAS BIBLIOGRÁFICAS}

Adegbile, J.A. y Adeyemi B.A. (2008). Enhancing quality assurance through teacher's effectiveness. Educational Research and Review, 3(2), 61-65.

Borich, G. (2009). Effective Teaching Methods. Trenton, NJ: Merrill.

Brookhart, S.M. (2009). Assessment and Examinations. En L.J. Saha y A.G. Dworkin (Eds.), International Handbook of Research on Teachers and Teaching (pp. 723-739). Nueva York: Springer.

Carreras, M. R., Guil, R. y Mestre (1999). Estilo motivacional asociado a percepciones positivas de eficacia docente. Cadiz: Rodin-UCA

Concha, C. (1996). Estudio de 32 escuelas de alta vulnerabilidad socioeconómica y altos resultados de aprendizaje. Santiago de Chile: Ministerio de Educación.

Cooper, H. (2007). The battle over homework. Thousand Oaks, CA: Corwin Press.

Cornejo, R. y Redondo, J.M. (2007). Variables y factores asociados al aprendizaje escolar. Estudios Pedagógicos, 33(2), 155-175.

Cueto, S., Ramírez, C., León, J. y Pain, O. (2002). Oportunidades de aprendizaje y rendimiento en matemática en una muestra de estudiantes de sexto grado de primaria de Lima. Lima: GRADE.

Darling-Hammond, L. (2000). Teacher quality and student achievement. Education Policy Analysis Archives, 8(1), 5-26.

Duarte, J., Bos, M.S. y Moreno, M. (2011). Los docentes, las escuelas y los aprendizajes escolares en América Latina: Un estudio regional usando la base de datos del SERCE. Washington, DC: Banco Internacional de Desarrollo.

Fernández Aguerre, T. (2004). De las escuelas eficaces a las reformas educativas de segunda generación. Estudios Sociológicos, 22(65), 377-408.

Gamoran, A., Porter, A.C., Smithson, J. y White, P.A. (1997). Upgrading high school mathematics instruction. Educational Evaluation and Policy Analysis, 19(4), 325-338.

Gran, L.W., Hindman, J. y Stronge, J.H. (2010). Planning, Instruction, and Assessment: Effective Teaching Practices. Nueva York: Eye on Education.

Hernández-Castilla, R., Murillo, F.J. y Martínez-Garrido, C. (2014). Factores de ineficacia escolar. REICE. Revista Iberoamericana sobre Calidad, Eficacia y Cambio en Educación, 12(1), 103-118.

Hunt, G.H., Wiseman, D.G. y Touzel, T.J. (2009). Effective teaching: preparation and implementation. Springfield, Il: Charles C Thomas.

Killen, R. (2006). Effective teaching strategies. Sidney: Thomsom.

LLECE (2001). Informe técnico. Primer Estudio Internacional Comparativo sobre Lenguaje, Matemática y factores asociados, para alumnos del tercer y cuarto grado de la educación básica. Santiago de Chile: UNESCO.

López, M. (2006). Todo el que llega aquí se contagia: el éxito escolar. En F.J. Murillo (Ed.), Estudios sobre eficacia escolar en Iberoamérica: 15 buenas investigaciones (pp. 261-286). Bogotá: Convenio Andrés Bello.

López, G., Assael, G. y Neuman, E. (1984). La cultura escolar ¿ responsable del fracaso? Estudio etnográfico en dos escuelas urbano populares. Santiago de Chile: PIIE.

Marsh, H.W. (1988). Self Description-Questionnaire I. SDQ-I manual and research monograph. San Antonio, TX: The Psychological Corporation.

Marsh, H.W., Craven, R.G. y Debus, R.L. (1991). Self-concepts of young children aged 5 to 8: Their measurement and multidimensional structure. Journal of Educational Psychology, 83, 377-392.

Martínez-Garrido, C. (2015). Investigación sobre Enseñanza Eficaz. Un estudio multinivel para Iberoamérica. Tesis doctoral. Madrid: UAM Ediciones.

Marzano, R.J. (2003). What Works in Schools: Translating Research into Action. Alexandria, VA: ASCD.

Muijs, D. y Reynolds, D. (2001). Effective Teaching. Evidence and Practice. Londres: Sage. 
Muñoz-Izquierdo, C., Rodríguez, P. G., De Cepeda, P. R. y Borrani, C. (1979). El síndrome del atraso escolar y el abandono del sistema educativo. Revista Latinoamericana de Estudios Educativos, 20(3), 221-285.

Murillo, F. J. (2006). Un estudio multinivel sobre los efectos escolares y los factores de eficacia de los centros docentes de primaria en España. Estudios sobre eficacia escolar en Iberoamérica, $15,345-372$.

Murillo, F.J. (2007a). School Effectiveness Research in Latin America. En T. Townsend (Ed.), International Handbook of School Effectiveness and Improvement (pp. 75-92). Nueva York: Springer.

Murillo, F.J. (Coord.) (2007b). Investigación Iberoamericana sobre Eficacia Escolar. Bogotá: Convenio Andrés Bello.

Murillo, F.J. y Martínez-Garrido, C. (2012). Las condiciones ambientales en las aulas de primaria en Iberoamérica y su relación con el desempeño académico. Archivos Analíticos de Políticas Educativas, 20(18), 1-24.

Murillo, F.J. y Martínez-Garrido, C. (2013). Homework Influence on academic performance. A study of Iberoamerican students of Primary Education. Revista de Psicodidáctica, 18(1), 157-171.

Murillo, F.J., Martínez-Garrido, C. y Hernández-Castilla, R. (2011). Decálogo para una enseñanza eficaz. REICE. Revista Iberoamericana sobre Calidad, Eficacia y Cambio en Educación, 9(1), $6-27$.

Murillo, J., Martínez-Garrido, C. e Hidalgo (2014). Incidencia de la forma de evaluar los docentes de Educación Primaria en el rendimiento de los estudiantes en España. ESE- Estudios sobre Educación, 27, 91-113.

Orlich, D.C., Harder, R.J., Callahan, R.C., Trevisan, M.S. y Brown, A.H. (2010). Teaching strategies: a guide to effective instruction. Boston, MA: Wadsworth.

Román, M. (2003). ¿Por qué los docentes no pueden desarrollar procesos de enseñanza aprendizaje de calidad en contextos sociales vulnerables? Persona y Sociedad, 17(1), 113-128.

Román, M. y Murillo, F.J. (2011). América Latina: Violencia entre estudiantes y desempeño escolar. Revista CEPAL, 104, 37-54.

Rubie-Davies, C.M. (2010). Teacher Expectations and Labeling. En L.J. Saha y A.G. Dworkin (Eds.), International Handbook of Research on Teachers and Teaching (pp. 695-707). Nueva York: Springer.

Sancho, A. (2006). La eficacia en América Latina: mitos y realidades. En F.J. Murillo (Coord.). Estudios sobre eficacia escolar en iberoamérica. 15 buenas investigaciones (pp. 411-458). Bogotá: Convenio Andrés Bello.

Schiefelbein, E., Vélez, E. y Valenzuela, J. (1997). Factores que Afectan el rendimiento Académico en la Educación Primaria. Revisión de la Literatura de América Latina y el Caribe. Washington, DC: Banco Mundial.

Thapa, A., Cohen, J. Guffey, S. y Higgins-D'Alessandro, A. (2013). A Review of School Climate Research. Review of Educational Research, 8(3), 357-385.

Thijs J. y Verkuyterm, M. (2009). Students' anticipated situational engagement: the roles of teach-in behavior, personal engagement and gender. The Journal of Genetic Psychology: Research and Theory on Human Developmeny, 170(3), 268-286.

Trautwein, U. y Köller, O. (2003). Time investment does not always pay off: the role of self-regulatory strategies in homework execution. Zeitschrift fur Padagogische Psychologie, 17, 199-209.

Villa, A. (1983). Multidimensionalidad del modelo de profesor ideal y condicionantes estructurales que lo determinan. Bilbao: ICE de la Universidad de Deusto.

Wittrock, M. (1986). Handbook of Research Teaching. Nueva York: MacMillan. 
